\title{
Time to blood cultures positivity of microorganisms using a continuous-monitoring automated blood cultures system
}

\author{
Pajaree Krisanapan, Romanee Chaiwarith*
}

\begin{abstract}
Background: Previous studies showed that clinically significant pathogens were detected within 3 days of incubation using a continuous monitoring automated blood culture instrument.

Objectives: To determine time to blood culture positivity (TTP) of microorganisms using a BD BACTEC ${ }^{\text {TM FX. }}$

Methods: A cross-sectional study was conducted at Maharaj Nakorn Chiang Mai Hospital, Thailand from October to November 2014.

Results: One-hundred and eighty-one patients with 195 episodes of infection and 436 cultures were included in the analysis. Among 181 patients, 55.2\% were male and the median age was 61 years (interquartile range (IQR) 50, 76). Of the 195 episodes of infections, the most common source was genitourinary tract (15.4\%). Overall, the median TTP was 17 hours (IQR 11.5, 24.5), the shortest TTP was observed in Streptococcus agalactiae. Four-hundred and seventyeight cultures (97.6\%) and all (100\%) were detected at 3 days and 5 days of incubation. Factors associated with TTP $\leq 24$ hour were blood drawn from patients who had hematologic malignancy (odds ratio (OR) 9.6, 95\% confidence interval (CI) 1.2, 74.3, $P=0.030$ ), endocarditis and vascular infection (OR 8.7, 95\% CI 1.1, 67.2, $P=0.038$ ), thrombocytopenia (OR 2.4, 95\% CI 1.3, 4.4, $P=0.004$ ), clinical of systemic inflammatory response syndrome (SIRS) (OR 2.3, 95\% CI 1.2, 4.5, $P=0.014$ ), and not receiving antimicrobials within 72 hours before cultures taken (OR 2.2, $95 \%$ CI $1.4,3.6, P<0.001)$
\end{abstract}

Conclusions: TTP varied depends upon the pathogens and clinical settings. However, bacteria were isolated from almost, but not all of the blood cultures within 3 days of incubation.

Keywords: bacteremia; blood culture; fungemia

Blood culture remains the gold standard for diagnosis of bloodstream infection. Time to blood culture positivity (TTP) depends upon the type of bacteria, whether they are true pathogens or contaminants, the severity of sepsis, and the underlying diseases [1-5]. Previous studies have shown that clinically significant pathogens were detected within 3 days of incubation using continuous monitoring automated blood culture instruments, missing 3-5\%, and raised the question whether 3 days are enough for blood culture incubation [2, 5-12].

Currently, a continuous automated blood culture instrument BD BACTEC ${ }^{\text {TM }}$ FX (Becton, Dickinson and Company, Franklin Lakes, New Jersey) is used in our institution,

*Correspondence to: Romanee Chaiwarith, Department of Medicine, Faculty of Medicine, Chiang Mai University, Chiang Mai 50200, Thailand, e-mail: romanee.c@cmu.ac.th

Department of Medicine, Faculty of Medicine, Chiang Mai University, Chiang Mai 50200, Thailand

O Open Access. ๑ 2019 Krisanapan and Chaiwarith, published by Sciendo. (c) BY-NC-ND This work is licensed under the Creative Commons Attribution NonCommercial-NoDerivatives 4.0 License. 
Maharaj Nakorn Chiang Mai Hospital. The 5-day-incubation period is recommended as per the manufacturer instruction before reporting negative results [13].

The primary objective of this study was to determine the TTP of microorganisms growing in blood cultures drawn from adult patients. The secondary objectives were to (1) determine the cumulative probability of TTP of microorganisms, (2) the TTP among patients with and without clinical of systemic inflammatory response syndrome (SIRS), and (3) determine factors associated with the rapid growth of microorganisms.

\section{Materials and methods}

\section{Study setting, design, and population}

This retrospective study was approved by the Research Ethics Committee Faculty of Medicine, Chiang Mai University (certificate of approval no. 272/2014).

A cross-sectional study was conducted among patients receiving care at Maharaj Nakorn Chiang Mai Hospital from October 1 to November 30, 2014. All patients who met the following criteria were consecutively enrolled: (1) age $\geq 15$ years old, (2) had blood cultures drawn at the emergency department, outpatient department, and/or inpatient units, (3) blood culture grew organisms, and (4) medical records were available for review.

\section{Data collection}

Demographic (e.g. age, sex, underlying diseases) and clinical characteristics including medical services, clinical of SIRS, source of infection, prior antimicrobial uses, organisms grew from blood cultures, and TTP for each blood culture bottle were collected. Patients with multiple episodes of infection were counted only once for analysis of demographic characteristics, but counted as the number of the episode for analysis of clinical characteristics.

\section{Procedures for blood cultures}

Blood culture was obtained by trained health care personnel using $2 \%$ alcoholic chlorhexidine or $75 \%$ alcohol followed by $10 \%$ povidone-iodine as skin antisepsis for peripheral venipuncture. The aseptic technique was also performed in blood obtained from central venous/arterial catheters. At least $10 \mathrm{~mL}$ of blood was obtained for each bottle and inoculated into aerobic blood culture bottle using BD BACTEC ${ }^{\text {TM }}$ Plus
Aerobic/F culture bottles for bacteria processing on the $\mathrm{BD}$ BACTEC FX ${ }^{\mathrm{TM}}$ (Becton, Dickinson and Company, Franklin Lakes, New Jersey) at the central diagnostic laboratory, Maharaj Nakorn Chiang Mai Hospital. Anaerobic blood cultures were not routinely performed in our hospital. Each patient who had $\geq 2$ blood culture bottles drawn should have been drawn at least 30 minutes apart, except for emergency conditions in which two blood cultures were drawn at the same time but from different venipuncture sites. Positive blood culture was reported immediately when the organism was detected, whereas negative blood culture was reported when no organism growth was observed after incubation for 5 days.

\section{Definitions}

Episode of infection was defined as the first positive blood culture in a series and judged by investigators according to clinical information.

TTP is defined as the beginning time of the blood culture incubation until the positive result was detected by the automated system. It is the standard practice in our hospital that blood culture bottle must then be sent to the central diagnostic laboratory as soon as possible, and usually within 30 minutes after blood drawn.

True pathogen is considered for Staphylococcus aureus, Streptococcus pneumoniae, Streptococcus pyogenes, Streptococcus agalactiae, Listeria monocytogenes, Neisseria meningitidis, Neisseria gonorrhoeae, Haemophilus influenzae, Escherichia coli and other enterobacteriaceae, Pseudomonas aeruginosa, members of the Bacteroides fragilis group, all Candida species, and Cryptococcus neoformans [14-16].

Contaminant blood culture is considered if (1) 1 of 1 blood culture obtained or 1 of $\geq 2$ blood cultures obtained grew coagulase-negative staphylococci, Corynebacterium spp., Micrococcus spp., Bacillus spp. not anthracis, or viridans group streptococci or (2) $\geq 2$ blood cultures obtained within 48 hours grew coagulase-negative staphylococci, Corynebacterium spp., Micrococcus spp., Bacillus spp. not anthracis, or viridans group streptococci, in which the isolates were different, that is, different antibiograms [17]. However, in the presence of central venous or arterial catheters, the isolation of coagulase-negative staphylococci might be considered as a true pathogen by taking into account of the following factors: clinical manifestations, leukocyte count, imaging results, clinical course, response to therapy, and the absence or presence of other sites of infection.

Multiple isolates blood culture is defined if $>1$ microorganism was isolated from the same bottle or if two populations of the same microorganism with different antibiotic 
susceptibility patterns were identified [5]. Only cultures from which a single organism was recovered were used to analyze time-to-positivity.

SIRS, sepsis, and septic shock were followed those of Levy et al. and has been described elsewhere [18].

\section{Statistical analysis}

Data were presented in mean $\pm \mathrm{SD}$, median and IQR, number (\%) as appropriate. Comparisons between groups were compared using Student's $t$-test or Mann Whitney $U$ test for continuous data, and $\chi^{2}$ test and Fisher's exact test for categorical data. TTP between groups was analyzed using Kaplan-Meier estimates and log rank tests. Factors associated with TTP within 24 hours were tested in univariate models. Factors with the $P<0.10$ from univariate analysis were then tested in a multivariate logistic regression model using backward stepwise procedure. Statistical significance was set as two-tailed, with $P<0.05$. All statistical analyses were performed using Stata statistical software version 11.0 (StataCorp. 2009. Stata Statistical Software: Release 11. College Station, TX: StataCorp LP.).

\section{Results}

During the study period, there were 6,961 blood cultures taken and $635(9.1 \%)$ grew microorganisms. One-hundred and twenty-nine blood cultures were excluded as shown in Figure 1. There were 506 cultures contributed from 238 patients, 103 patients contributed 1 blood culture bottle;
88 patients contributed 2 blood culture bottles; 18 patients contributed 3 blood culture bottles; 11 patients contributed 4 blood culture bottles; 7 patients contributed 5 blood culture bottles; 6 patients contributed 6 blood culture bottles; 2 patients contributed 7 blood culture bottles; 2 patients contributed 10 blood culture bottles, and 1 patient contributed 24 blood culture bottles.

Seventy cultures (13.8\%) were classified as contaminant blood cultures. Therefore, only true infection comprised of 436 cultures from 181 patients with 195 episodes of infection was included in the analysis of demographic and clinical characteristics.

Among 181 patients, $100(55.2 \%)$ were male and the median age was 61 years (IQR 50, 76). The most common location where blood cultures were drawn was inpatient units (90.6\%), which comprised of mostly from medical units $(60.8 \%)$ and surgical units $(23.8 \%)$. One-fourth of patients were admitted in critical care units.

The common underlying diseases were malignancy (34.8\%), chronic kidney disease (12.7\%), and diabetes mellitus $(11.6 \%)$ as shown in Table 1.

\section{Episodes of infections}

There were 195 episodes of infection. The common clinical presentations were fever (73.3\%), dyspnea (19\%), and gastrointestinal symptoms (18\%). Genitourinary tract infection was the most common source of bloodstream infection (15.4\%), followed by gastrointestinal and hepatobiliary tract infection (14.9\%) and catheter-related infection (12.8\%). Blood cultures were taken while receiving antimicrobials in 55 episodes

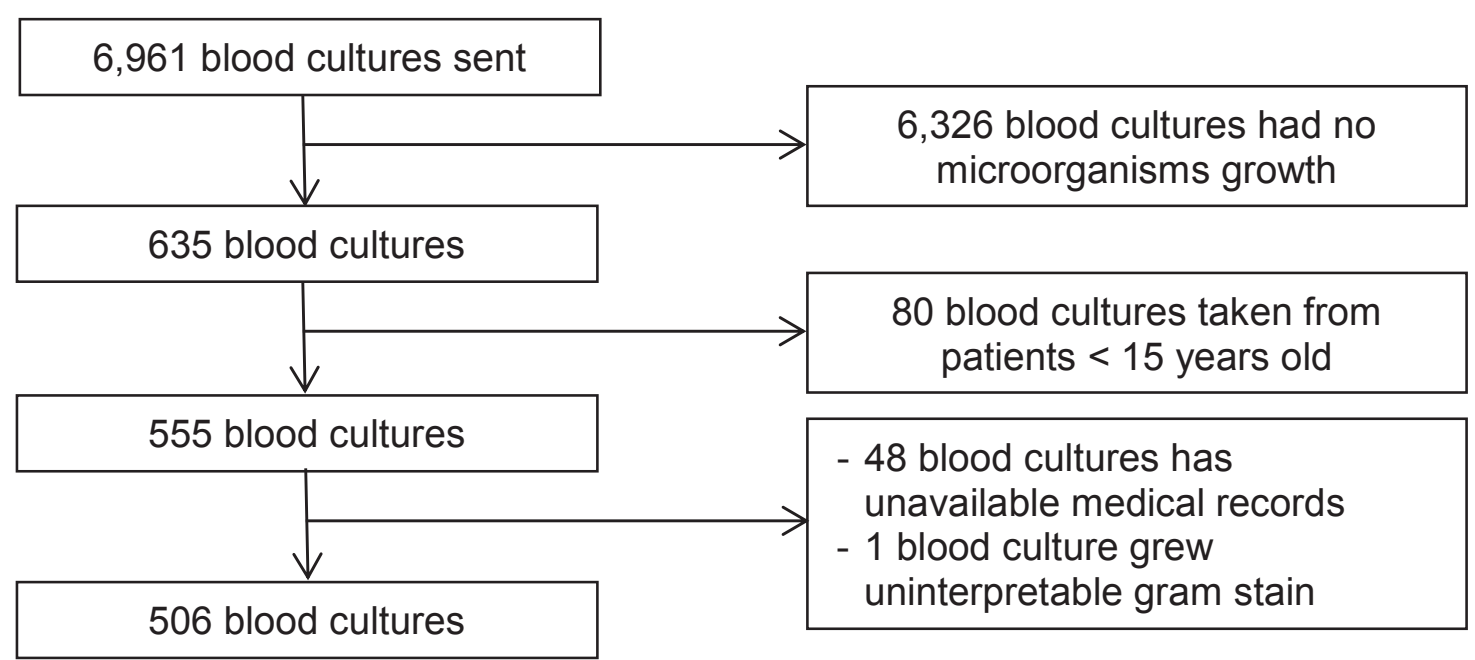

Figure 1. Blood cultures recruited for this study. 
Table 1. Demographic characteristics of 181 patients whose blood cultures grew true pathogens

\begin{tabular}{|c|c|}
\hline Characteristics & $\begin{array}{l}\text { Number (\%) } \\
\quad \mathrm{N}=\mathbf{1 8 1}\end{array}$ \\
\hline Male & $100(55.2)$ \\
\hline Age (years) (median, IQR) & $61(50,76)$ \\
\hline \multicolumn{2}{|l|}{ Location where blood cultures were drawn } \\
\hline Inpatient units & $164(90.6)$ \\
\hline Critical care units & $42(23.2)$ \\
\hline Noncritical care units & $122(67.4)$ \\
\hline Medicine & $110(60.8)$ \\
\hline Surgery & $43(23.8)$ \\
\hline Orthopedics & $5(2.8)$ \\
\hline Obstetrics and gynecology & $3(1.7)$ \\
\hline Others & $3(1.7)$ \\
\hline Outpatient department & $10(5.5)$ \\
\hline Emergency department & $7(3.9)$ \\
\hline Underlying diseases & $145(80.1)$ \\
\hline Malignancy & $63(34.8)$ \\
\hline Hematologic malignancy & $18(9.9)$ \\
\hline Solid organ malignancy & $45(24.9)$ \\
\hline $\begin{array}{l}\text { Advanced chronic kidney disease } \\
\left(\mathrm{GFR}<30 \mathrm{~mL} / \mathrm{min} / 1.73 \mathrm{~m}^{2}\right)\end{array}$ & $23(12.7)$ \\
\hline Diabetes & $21(11.6)$ \\
\hline Disease result in neurocognitive impairment & $14(7.7)$ \\
\hline Symptomatic heart failure or ejection fraction $<40 \%$ & $13(7.2)$ \\
\hline Cirrhosis & $12(6.6)$ \\
\hline Hydronephrosis & $8(4.4)$ \\
\hline HIV infection & $7(3.9)$ \\
\hline Chronic obstructive lung disease & $5(2.8)$ \\
\hline Gastrointestinal fistula & $5(2.8)$ \\
\hline Rheumatologic disease & $3(1.7)$ \\
\hline Post splenectomy & $3(1.7)$ \\
\hline Post organ transplant & $3(1.7)$ \\
\hline Aplastic anemia & $2(1.1)$ \\
\hline Adult onset immunodeficiency syndrome & $1(0.6)$ \\
\hline Received immunosuppressive agents & $34(18.8)$ \\
\hline
\end{tabular}

GFR, glomerular filtration rate; HIV, human immunodeficiency virus; $\mathrm{IQR}$, interquartile range

(28.2\%). Clinical characteristics of 195 episodes of infections are shown in Table 2 and clinical parameters are shown in Table 3.

\section{Time to blood cultures positivity}

Among 506 blood cultures, 490 cultures grew single microorganism, 16 cultures contained 2 microorganisms. All 70 contaminant blood cultures contained Gram-positive bacteria which included coagulase-negative staphylococci (38 cultures), Bacillus spp. (22 cultures), Corynebacterium spp. (7 cultures),
Table 2. Clinical characteristics of 195 episodes of infections

\begin{tabular}{lc}
\hline Characteristics & Number (\%) \\
& N $=\mathbf{1 9 5}$ \\
\hline Clinical presentations & \\
Fever & $143(73.3)$ \\
Dyspnea & $37(19.0)$ \\
Abdominal pain, diarrhea, nausea, vomiting & $35(18.0)$ \\
Altered mental status & $30(15.4)$ \\
Dysuria, flank pain, or cloudy urine & $14(7.2)$ \\
Source of infection & \\
Genitourinary tract & $30(15.4)$ \\
Gastrointestinal and hepatobiliary tract & $29(14.9)$ \\
Central venous catheter related & $25(12.8)$ \\
Respiratory tract & $15(7.7)$ \\
Skin, bone, joint, and soft tissue & $12(6.2)$ \\
Endocarditis and vascular system & $8(4.1)$ \\
Unidentified & $72(36.9)$ \\
Prior antimicrobial uses & $\mathbf{6 3 ( 3 2 . 3 )}$ \\
Type of antimicrobials & \\
Antibiotics & $62(31.8)$ \\
Cephalosporins & $16(8.2)$ \\
Carbapenems & $14(7.2)$ \\
Quinolones & $14(7.2)$ \\
Vancomycin & $13(6.7)$ \\
Metronidazole & $12(6.2)$ \\
Penicillin & $9(4.6)$ \\
Trimethoprim/sulfamethoxazole & $5(2.6)$ \\
Colistin & $3(1.5)$ \\
Macrolides & $1(0.5)$ \\
Aminoglycosides & $1(0.5)$ \\
Others & $3(1.5)$ \\
Current use of antimicrobials at the time blood & \\
\hline & $\mathbf{5 5}(4.6)$ \\
\hline & \\
\hline & \\
Cultures were taken & \\
\hline
\end{tabular}

and Micrococcus spp. (3 cultures). The list of microorganisms and TTP for each microorganism is shown in Table 4. Sixteen multiple isolates blood cultures were excluded from the analysis of TTP.

Overall, the median TTP was 17 hours (IQR 11.5, 24.5), the medians TTP for Gram-positive and Gram-negative bacteria were 17.5 (IQR 12.5, 23) and 14 hours (IQR 10.5, 20), respectively $(P=0.004)$. The median TTP for yeast was 27.5 hours (IQR 22, 38). S. agalactiae had the shortest TTP, followed by Vibrio vulnificus, S. pneumoniae, and Streptococcus bovis with the medians TTP of 5.5 (IQR 5.5, 6), 8.5 (IQR $8.5,8.5), 10$ (IQR 5,12$)$, and $10(8.5,12.5)$ hours, respectively.

Among contaminant blood cultures, medians TTP are shown in Table 5. The medians TTP for true pathogens and contaminants were 16 (IQR 11.5, 23), and 23 (IQR 18.5, 28) hours, respectively $(P<0.001)$. The median TTP for 
Table 3. Clinical parameter of 195 episodes of infections

\begin{tabular}{|c|c|c|}
\hline Sepsis parameters & $\begin{array}{c}\text { Total } \\
\text { number of } \\
\text { available } \\
\text { data }\end{array}$ & Number (\%) \\
\hline Body temperature $<36$ or $>38.3^{\circ} \mathrm{C}$ & 191 & $134(70.2)$ \\
\hline Heart rate $>90$ beat $/ \mathrm{min}$ & 191 & $168(88.0)$ \\
\hline Respiratory rate $>20 / \mathrm{min}$ & 190 & $105(55.3)$ \\
\hline Hypotension or shock & 191 & $56(29.3)$ \\
\hline Altered mental status & 191 & $30(15.7)$ \\
\hline Acute lung injury & 153 & $27(17.6)$ \\
\hline Decrease urine output & 141 & $22(15.6)$ \\
\hline $\begin{array}{l}\text { Creatinine }>2.0 \mathrm{mg} / \mathrm{dL} \text { or increase } \\
>0.5 \mathrm{mg} / \mathrm{dL}\end{array}$ & 162 & $43(26.5)$ \\
\hline Lactate level (mmol/L) (median, IQR) & 95 & $3.0(1.7,6.2)$ \\
\hline Lactate level $>1.0 \mathrm{mmol} / \mathrm{L}$ & 95 & $91(95.8)$ \\
\hline $\begin{array}{l}\text { White blood cells }\left(\times 10^{3} \text { cells } / \mathrm{mm}^{3} \text { ) }\right. \\
\text { (median, IQR) }\end{array}$ & 195 & $12.5(7.7,17)$ \\
\hline $\begin{array}{l}\text { White blood cells }<4,000 \text { or }>12,000 \\
\text { cells } / \mathrm{mm}^{3}\end{array}$ & 195 & $136(69.7)$ \\
\hline $\begin{array}{l}\text { Platelet count }\left(\times 10^{3} \mathrm{per} / \mathrm{mm}^{3}\right) \\
\text { (median, IQR) }\end{array}$ & 194 & $161(70,261)$ \\
\hline Platelet count $<100,000 / \mathrm{mm}^{3}$ & 194 & $71(36.6)$ \\
\hline $\begin{array}{l}\text { Clinical systemic inflammatory } \\
\text { response syndrome }\end{array}$ & 191 & $172(90.0)$ \\
\hline
\end{tabular}

$\mathrm{IQR}$, interquartile range

Table 4. List of microorganisms and time to positivity

\begin{tabular}{lcc}
\hline Microorganisms & $\begin{array}{c}\text { Number of } \\
\text { blood } \\
\text { cultures (\%) }\end{array}$ & $\begin{array}{c}\text { Median time to } \\
\text { positivity (hours) } \\
\text { (IQR) }\end{array}$ \\
\hline Overall & $490(100)$ & $17(11.5,24.5)$ \\
Bacteria & $433(88.4)$ & $15.5(11.5,22)$ \\
Gram-positive bacteria & $214(43.7)$ & $17.5(12.5,23)$ \\
$\quad$ Staphylococci & $123(25.1)$ & $19(15,23.5)$ \\
$\quad$ Coagulase-negative & $67(13.7)$ & $20.5(18,25)$ \\
Staphylococci & & \\
Staphylococcus aureus & $56(11.4)$ & $15.5(12.5,20.25)$ \\
Streptococci & $50(10.2)$ & $12.25(9.5,15.5)$ \\
$\quad$ Streptococcus bovis & $15(3.1)$ & $10(8.5,12.5)$ \\
Streptococcus pneumoniae & $5(1.0)$ & $10(5,12)$ \\
Streptococcus pyogenes & $5(1.0)$ & $11(10,12)$ \\
Streptococcus agalactiae & $5(1.0)$ & $5.5(5.5,6)$ \\
$\quad$ Other Streptococci & $20(4.1)$ & $15.5(14,17.25)$ \\
Bacillus spp. & $22(4.5)$ & $17.25(12.5,25.5)$ \\
Enterococcus faecium & $7(1.4)$ & $17.5(16.5,19)$ \\
Corynebacterium spp. & $7(1.4)$ & $26.5(23,58.5)$ \\
Micrococcus spp. & $3(0.6)$ & $87(57,90)$ \\
Listeria monocytogenes & $2(0.4)$ & $19.5(19.5,19.5)$ \\
\hline
\end{tabular}

(Continued)
Table 4. Continued

\begin{tabular}{|c|c|c|}
\hline Microorganisms & $\begin{array}{c}\text { Number of } \\
\text { blood } \\
\text { cultures (\%) }\end{array}$ & $\begin{array}{c}\text { Median time to } \\
\text { positivity (hours) } \\
\text { (IQR) }\end{array}$ \\
\hline Gram-negative bacteria & $219(44.7)$ & $14(10.5,20)$ \\
\hline Escherichia coli & $55(11.2)$ & $12(10.5,15)$ \\
\hline Klebsiella pneumoniae & $35(7.1)$ & $10.5(9.5,13)$ \\
\hline Pseudomonas aeruginosa & $28(5.7)$ & $21(17.5,25.25)$ \\
\hline Acinetobacter baumannii & $17(3.5)$ & $11(10,12.5)$ \\
\hline $\begin{array}{l}\text { Aeromonas sobria and } \\
\text { A.hydrophilla }\end{array}$ & $17(3.5)$ & $10.5(9,13)$ \\
\hline Salmonella spp. & $14(2.9)$ & $12.5(10.5,16.5)$ \\
\hline Burkholderia cepacia & $10(2.0)$ & $22.25(20,39)$ \\
\hline $\begin{array}{l}\text { Enterobacter cloacae and } \\
\text { Enterobactor spp. }\end{array}$ & $8(2.0)$ & $22.5(14.25,54)$ \\
\hline $\begin{array}{l}\text { Stenotrophomonas } \\
\text { maltophilia }\end{array}$ & $8(1.6)$ & $18.5(16.25,26.5)$ \\
\hline Proteus mirabilis & $5(1.0)$ & $14(13.5,17)$ \\
\hline Elizabethkingia meningoseptica & $4(0.8)$ & $13.75(12.25,15)$ \\
\hline Citrobacter freundii and C. koseri & $3(0.6)$ & $10.5(10.5,13)$ \\
\hline Serratia marcescens & $3(0.6)$ & $18(17,29)$ \\
\hline Neisseria gonorrhoeae & $2(0.4)$ & $29.5(29,30)$ \\
\hline Vibrio vulnificus & $2(0.4)$ & $8.5(8.5,8.5)$ \\
\hline Campylobacter spp. & $1(0.2)$ & 45 \\
\hline $\begin{array}{l}\text { Unidentified Gram-negative } \\
\text { bacilli }\end{array}$ & $7(1.4)$ & $32(28.5,43)$ \\
\hline Yeast and other fungi & $57(11.6)$ & $27.5(22,38)$ \\
\hline Candida spp. & $55(11.2)$ & $27(22,36.5)$ \\
\hline Candida tropicalis & $14(2.9)$ & $19.8(19,22)$ \\
\hline Candida albicans & $10(2.0)$ & $40.5(34,54)$ \\
\hline Candida parapsilosis & $3(0.6)$ & $41.5(26.5,87.5)$ \\
\hline Candida guilliermondii & $3(0.6)$ & $28(26,30)$ \\
\hline Candida glabrata & $3(0.6)$ & $61.5(60.5,71)$ \\
\hline Unidentified Candida spp. & $22(4.5)$ & $27.8(25.5,31)$ \\
\hline $\begin{array}{l}\text { Talaromyces (Penicillium) } \\
\text { marneffei }\end{array}$ & $1(0.2)$ & 110.5 \\
\hline $\begin{array}{l}\text { Unidentified dematiaceous } \\
\text { fungi }\end{array}$ & $1(0.2)$ & 82.5 \\
\hline
\end{tabular}

$\mathrm{IQR}$, interquartile range

coagulase-negative staphylococci which were true pathogens and contaminants were $18.5(16.5,20.5)$ and $22(19.5,25.5)$ hours, respectively.

Three-hundred and sixty-seven (74.9\%) blood cultures grew within 1 day, 462 (94.3\%) cultures grew within 2 days, $478(97.6 \%)$ cultures grew within 3 days, $489(99.8 \%)$ cultures grew within 4 days, and all $490(100 \%)$ cultures grew within 5 days. Microorganisms with TTP $>3$ days were Micrococcus spp. (2 cultures), Microaerophillic streptococci (1 culture), Corynebacterium diphtheria (1 culture), Burkholderia cepacia (2 cultures), E. coli (1 culture), E. cloacae (1 culture), Candida albicans (1 culture), C. parapsilosis (1 culture), unidentified 
Table 5. Median time to positivity comparing true pathogens and contaminant blood cultures

\begin{tabular}{|c|c|c|c|c|c|}
\hline \multirow[t]{2}{*}{ Organism } & \multirow{2}{*}{$\begin{array}{c}\text { Total } \\
\text { number }\end{array}$} & \multicolumn{2}{|c|}{ True pathogen } & \multicolumn{2}{|c|}{ Contaminant blood cultures } \\
\hline & & Number (\%) & TTP (IQR) & Number (\%) & TTP (IQR) \\
\hline Overall & 490 & $420(85.7)$ & $16(11.5,23)$ & $70(14.3)$ & $23(18.5,28)$ \\
\hline Gram-positive bacteria & 214 & $144(67.3)$ & $15.5(12,19)$ & $70(32.7)$ & $23(18.5,28)$ \\
\hline Coagulase-negative staphylococci & 67 & $29(43.3)$ & $18.5(16.5,20.5)$ & $38(56.7)$ & $22(19.5,25.5)$ \\
\hline Bacillus spp. & 22 & 0 & - & $22(100)$ & $17.25(12.5,25.5)$ \\
\hline Corynebacterium spp. & 7 & 0 & - & $7(100)$ & $26.5(23,58.5)$ \\
\hline Micrococcus spp. & 3 & 0 & - & $3(100)$ & $87(57,90)$ \\
\hline
\end{tabular}

$\mathrm{IQR}$, interquartile range; TTP, time to blood culture positivity

Table 6. The cumulative median time to positivity for particular microorganisms

\begin{tabular}{|c|c|c|c|c|c|c|c|}
\hline \multirow[t]{2}{*}{ Microorganisms } & \multirow[t]{2}{*}{$\mathbf{N}$} & \multicolumn{6}{|c|}{ Number (\%) of organisms recovered on hours } \\
\hline & & $\leq 12$ & $\leq 24$ & $\leq 48$ & $\leq 72$ & $\leq 96$ & $\leq 120$ \\
\hline Overall & 490 & $142(29)$ & $367(75)$ & $462(94.3)$ & $478(97.6)$ & $489(99.8)$ & $490(100)$ \\
\hline Bacteria & 433 & $141(32.6)$ & $347(80.1)$ & $415(95.8)$ & $425(98.2)$ & $433(100)$ & $433(100)$ \\
\hline Gram-positive bacteria & 214 & $49(22.9)$ & $166(77.6)$ & $202(94.4)$ & $210(98.1)$ & $214(100)$ & $214(100)$ \\
\hline Staphylococci & 123 & $18(14.6)$ & $94(76.4)$ & $119(96.8)$ & $123(100)$ & $123(100)$ & $123(100)$ \\
\hline Coagulase-negative staphylococci & 67 & $5(7.5)$ & $48(71.6)$ & $65(97)$ & $67(100)$ & $67(100)$ & $67(100)$ \\
\hline Staphylococcus aureus & 56 & $13(23.2)$ & $46(82.1)$ & $54(96.4)$ & $56(100)$ & $56(100)$ & $56(100)$ \\
\hline Streptococci & 50 & $25(0.5)$ & $46(92)$ & $48(96)$ & $49(98)$ & $50(100)$ & $50(100)$ \\
\hline True pathogens & 144 & $42(28.2)$ & $124(86.1)$ & $139(96.5)$ & $143(99.3)$ & $144(100)$ & $144(100)$ \\
\hline Contaminants & 70 & $7(10)$ & $42(60)$ & $63(90)$ & $67(95.7)$ & $70(100)$ & 70 (100) \\
\hline Gram-negative bacteria & 219 & $92(42)$ & $181(82.6)$ & $213(97.3)$ & $215(98.2)$ & $219(100)$ & $219(100)$ \\
\hline Escherichia coli & 55 & $30(54.6)$ & $48(87.3)$ & $54(98.2)$ & $54(98.2)$ & $55(100)$ & $55(100)$ \\
\hline Klebsiella pneumoniae & 35 & $25(71.4)$ & $34(97.1)$ & $35(100)$ & $35(100)$ & $35(100)$ & $35(100)$ \\
\hline Pseudomonas aeruginosa & 28 & 0 & $20(71.4)$ & $28(100)$ & $28(100)$ & $28(100)$ & $28(100)$ \\
\hline Acinetobacter baumannii & 17 & $11(64.7)$ & $17(100)$ & $17(100)$ & $17(100)$ & $17(100)$ & $17(100)$ \\
\hline Aeromonas spp. & 17 & $12(70.6)$ & $16(94.1)$ & $17(100)$ & $17(100)$ & $17(100)$ & $17(100)$ \\
\hline Salmonella spp. & 14 & $6(42.9)$ & $13(92.9)$ & $14(100)$ & $14(100)$ & $14(100)$ & $14(100)$ \\
\hline Burkholderia spp. & 10 & 0 & $7(70)$ & $8(80)$ & $8(80)$ & $10(100)$ & $10(100)$ \\
\hline Yeast and other fungi & 57 & $1(1.8)$ & $20(35.1)$ & $47(82.5)$ & $53(93)$ & $56(98.3)$ & $57(100)$ \\
\hline
\end{tabular}

dematiaceous fungi (1 culture), and Talaromyces (Penicillium) marneffei (1 culture). The details of cumulative TTP for particular microorganisms are shown in Table 6. The median TTP for patients with and without clinical SIRS was 16 hours (IQR $11.5,23.5)$, and 21 hours $(16,39.5)$, respectively $(P=0.002)$.

\section{Factors associated with time to blood culture positivity within 24 hours}

Comparing characteristics between blood cultures grew within 24 hours and after 24 hours were shown in Table 7. Blood cultures grew $\leq 24$ hours were more likely drawn from patients who had hematologic malignancy, received immunosuppressive agents, altered mental status, endocarditis or vascular infection, clinical SIRS, high blood lactate, low white blood cell count, and low platelet count. On the other hand, blood culture grew $>24$ hours were more likely drawn from patients who had intravascular catheters, catheter-related infection, prior use of antimicrobials within 72 hours particularly carbapenems, vancomycin, and antifungals.

Multivariate analysis revealed that factors associated with TTP $\leq 24$ hours were blood cultures drawn from patients with hematologic malignancy (OR 9.6, 95\% CI 1.2, $74.3, P=0.030$ ), endocarditis and vascular infection (OR 8.7, $95 \%$ CI 1.1, 67.2, $P=0.038$ ), thrombocytopenia (OR 2.4, 95\% CI 1.3, 4.4, $P=0.004$ ), had clinical signs of SIRS (OR 2.3, $95 \%$ CI 1.2, 4.5, $P=0.014)$, and not receiving antimicrobials within 72 hours before cultures were taken (OR 2.2, 95\% CI $1.4,3.6, P<0.001)$. 
Table 7. Comparisons of characteristics of patients with positive blood culture within 24 hours and $>24$ hours

\begin{tabular}{|c|c|c|c|}
\hline \multirow[t]{2}{*}{ Characteristics } & \multicolumn{2}{|c|}{ Number of patients (\%) } & \multirow[t]{2}{*}{$P$} \\
\hline & TTP $\leq 24$ hours $(\mathrm{N}=367)$ & TTP $>24$ hours $(N=123)$ & \\
\hline Male & $206(56.1)$ & $75(61.0)$ & 0.400 \\
\hline Underlying diseases & $300(81.7)$ & $101(82.1)$ & 1.000 \\
\hline Malignancy & $134(36.5)$ & $32(26.0)$ & 0.037 \\
\hline Hematologic malignancy & $50(13.6)$ & $1(0.8)$ & $<0.001$ \\
\hline Solid organ malignancy & $84(22.9)$ & $31(25.2)$ & 0.624 \\
\hline Receiving immunosuppressive agents & $78(21.2)$ & $13(10.6)$ & 0.007 \\
\hline Diabetes mellitus & $37(10.1)$ & $9(7.3)$ & 0.475 \\
\hline Cirrhosis & $19(5.2)$ & $4(3.2)$ & 0.468 \\
\hline HIV infection & $13(3.5)$ & $2(1.6)$ & 0.376 \\
\hline Post splenectomy & $4(1.1)$ & $1(0.8)$ & 1.000 \\
\hline Post organ transplant & $6(1.6)$ & $3(2.4)$ & 0.698 \\
\hline \multicolumn{4}{|l|}{ Clinical presentations } \\
\hline Fever & $277(76.5)$ & $90(75.0)$ & 0.805 \\
\hline Dyspnea & $79(21.8)$ & $20(16.7)$ & 0.243 \\
\hline Altered mental status & $58(16.0)$ & $9(7.5)$ & 0.022 \\
\hline \multicolumn{4}{|l|}{ Source of infections } \\
\hline Genitourinary tract & $41(11.2)$ & $12(9.8)$ & 0.739 \\
\hline Gastrointestinal and hepatobiliary system & $44(12.0)$ & $21(17.1)$ & 0.167 \\
\hline Catheter related & $52(14.2)$ & $28(22.8)$ & 0.034 \\
\hline Respiratory tract & $23(6.3)$ & $4(3.2)$ & 0.258 \\
\hline Skin, bone, joint, and soft tissue & $21(5.7)$ & $5(4.1)$ & 0.643 \\
\hline Endocarditis and vascular system & $22(6.0)$ & $1(0.81)$ & 0.014 \\
\hline Central nervous system & $2(0.5)$ & 0 & 1.000 \\
\hline Prior antimicrobials uses within 72 hours & $129(35.6)$ & $64(53.3)$ & 0.001 \\
\hline Carbapenems & $35(9.5)$ & $22(17.9)$ & $<0.001$ \\
\hline Vancomycin & $23(6.3)$ & $25(20.3)$ & $<0.001$ \\
\hline Antifungals & $17(4.6)$ & $20(16.3)$ & $<0.001$ \\
\hline Current uses of antimicrobials at the time of blood culture taken & $115(32.5)$ & $63(51.2)$ & $<0.001$ \\
\hline Indwelling intravascular catheters & $114(31.1)$ & $55(44.7)$ & 0.008 \\
\hline Clinical systemic inflammatory response syndrome & $337(93.1)$ & $99(83.2)$ & 0.003 \\
\hline Body Temperature $<36^{\circ} \mathrm{C}$ or $>38.3^{\circ} \mathrm{C}$ & $246(68.3)$ & $75(63.6)$ & 0.367 \\
\hline Heart rate $>90$ beats $/ \mathrm{min}$ & $336(91.6)$ & $110(89.4)$ & 0.470 \\
\hline Tachypnea & $213(59.7)$ & $66(55.5)$ & 0.452 \\
\hline Hypotension or shock & $116(32.0)$ & $46(38.3)$ & 0.221 \\
\hline Altered mentation & $62(21.0)$ & $14(14.9)$ & 0.233 \\
\hline Acute lung injury & $51(17.7)$ & $14(14.3)$ & 0.532 \\
\hline Lactate $>3 \mathrm{mmol} / \mathrm{L}$ & $100(59.5)$ & $18(32.7)$ & 0.001 \\
\hline White blood cells $<4,000$ cells $/ \mathrm{mm}^{3}$ & $68(18.5)$ & $9(7.4)$ & 0.003 \\
\hline Platelet count $<100,000 / \mathrm{mm}^{3}$ & 127(34.6) & $24(19.7)$ & 0.002 \\
\hline Gram-positive bacteria & $166(47.8)$ & $48(55)$. & 0.228 \\
\hline Gram-negative bacteria & $181(52.2)$ & $38(44.2)$ & 0.228 \\
\hline
\end{tabular}

\section{Discussion}

Our study reported that the positive rate of blood cultures was $9.1 \%$. This number was not changed from the positive rate among 35,000 blood cultures tested in the year 2012 at our hospital (unpublished data). Previous studies reported that two-thirds of patients with sepsis had negative blood cultures
$[6,10,12]$, which could be due to local infection, inappropriate blood culture collection either timing or blood volume, or receiving antibiotics prior to blood culture collection $[10,12,16]$. In conjunction with these reasons, the low positive rate in our study might be due to the low probability of bacterial infections in the patients as blood cultures were almost always taken in patients admitted and had fever. The contamination 
rate in this study was $13.8 \%$, which is higher than previous reports of $<5 \%$ [19-21]. Previous studies reported that the contamination rate was high among blood cultures taken at the emergency department and not using alcoholic chlorhexidine as skin antisepsis [21, 22]. Although the contamination rate in this study was high among blood cultures taken at the emergency department (31.3\%) and outpatient department $(24.0 \%)$, these numbers contributed only $8 \%$ of all cultures (data not shown). In addition, $2 \%$ alcoholic chlorhexidine is recommended as skin antisepsis for adults in our hospital. This high contamination rate alerted the infection control team to emphasize healthcare personnel to comply with the guidelines for drawing blood cultures.

The median TTP among 490 cultures was 17 hours and $97.6 \%$ of microorganisms could be detected within 3 days. Microorganisms grew after 3 days included four fungi and eight bacteria. Among eight bacteria, three cultures were contaminants, three cultures were taken while receiving antibiotics (two for B. cepacia and one for E. coli), and two cultures were Microaerophillic streptococci and Enterobacter cloacae. The latter two cultures had another blood culture in the same set grew within 3 days. Four fungi were isolated after 3 days of incubation. If disseminated fungal infections were not suspected in these patients and blood cultures on medium mycolytic culture bottles for $\mathrm{TB} /$ fungus were not performed, these clinical isolates would be missed. McGowan et al. reported time to positivity in children who had positive blood cultures using the BacT/Alert Microbial Detection System (Organon Teknika, Durham, NC), almost all significant positive cultures were detected by 72 hours [5]. Bourbeau and Pohlman had also reported that $97.5 \%$ of all clinically significant isolates were detected in the first 3 days of incubation with standard BacT/Alert blood culture bottles [2]. The authors suggested that it might not be necessary to routinely incubate blood culture bottles for more than 3 days if a continuous monitoring automated blood culture system is used [2]. From our point of view, as $2.4 \%$ of cultures would be missed if cultures were incubated for 3 days, we would suggest incubating blood cultures per manufacturer instruction which is currently 5-day incubation period.

We further demonstrated that TTP among Gram-negative bacteria (14 hours, IQR 10.5, 20) was shorter than Grampositive bacteria (17.5 hours, IQR 12.5, 23). However, this significantly different in TTP might not have clinical relevance. Of note, S. agalactiae grew fastest among all clinical isolates (5.5 hours, IQR 5.5, 6). Comparisons with the TTP of the same organism, the TTP in our study was shorter than the study in pediatrics, e.g. 12.2 hours for $S$. agalactiae [5]. This could be explained by the volume of blood culture being obtained in pediatrics was smaller than in adults.
For contaminant blood cultures, the TTP was longer than true pathogens. However, there was also no difference in a positive rate between true pathogens and contaminants after 3 days of incubation. In addition, patients with clinical SIRS had shorter TTP than patients without clinical SIRS. However, the medians TTP in both groups were $<1$ day.

The TTP may depend on multiple factors, including the volume of blood inoculated, the time elapse from specimen collection to incubation, and the concentration of organism within the blood $[1,3,7]$. In our study, we demonstrated that factors associated with TTP $<24$ hours were an underlying disease of malignancy, endocarditis and vascular infection, thrombocytopenia, and clinical of SIRS. A study among patients with $E$. coli bacteremia also reported that rapid TTP ( $<7$ hours) was noted in patients who had active malignancies and neutropenia [1]. In addition, a study among patients with nontyphoidal salmonella bacteremia, rapid TTP $(<10$ hours) was associated with endovascular lesion and higher bacteremia score [3]. However, a study among children with S. pneumoniae bacteremia, no clinical or laboratory parameters was associated with the early growth of organisms [23]. Underlying disease of malignancy may represent impaired host immune status. Clinical of SIRS, and endocarditis and vascular infection may represent a high concentration of organism within the blood. Thrombocytopenia may represent indirect evidence of severe infection and high concentration of organism in the blood. Current or prior uses of antimicrobials within 3 days of blood cultures taken were associated with TTP $>24$ hours as antimicrobials may delay the growth of the organism.

This study has several limitations. First, although we have the guideline for taking blood for cultures, we believed that the guideline may not be well complied. One of the indirect evidence was a high rate of blood culture contamination. Therefore, the volume of blood culture in each bottle and time from drawing blood cultures to incubated instruments may not be accurate. Those factors may responsible for low positivity rate and longer TTP in those cultures. Second, the study period was too short. Given the finding that four fungi were missed if the incubation period was 3 days during only a 2 -month period, numbers of pathogens might be overlooked if the study period was extended.

\section{Conclusions}

TTP varied depends upon the pathogens and clinical settings. Although microorganisms were isolated from almost all of the blood cultures within 3 days of incubation, $2.4 \%$ of cultures would be missed. A 5-day incubation period per manufacturer instructions is still required. 
Author contributions. RC contributed to the conception and design of this study. PK acquired the data. Both authors analyzed and interpreted it. PK drafted the manuscript and RC critically revised it. Both authors approved the final version submitted for publication and take responsibility for statements made in the published article.

Acknowledgments. We would like to thank Mr. Banyong Kantawa for his help in providing microbiological data.

Conflict of interest statement. The authors have completed and submitted the International Committee of Medical Journal Editors Uniform Disclosure Form for Potential Conflicts of Interest. None of the authors disclose any conflict of interest.

\section{References}

[1] Bo SN, Bo J, Ning YZ, Zhao Y, Lu XL, Yang JY, et al. Relationship between time to positivity of blood culture with clinical characteristics and hospital mortality in patients with Escherichia coli bacteremia. Chin Med J (Engl). 2011; 124:330-4.

[2] Bourbeau PP, Pohlman JK. Three days of incubation may be sufficient for routine blood cultures with BacT/Alert FAN blood culture bottles. J Clin Microbiol. 2001; 39:2079-82.

[3] Lin HW, Hsu HS, Huang YT, Yang CJ, Hsu MS, Liao CH. Time to positivity in blood cultures of adults with nontyphoidal Salmonella bacteremia. J Microbiol Immunol Infect. 2014; 49:417-23.

[4] Alvarez R, Vinas-Castillo L, Lepe-Jimenez JA, GarciaCabrera E, Cisneros-Herreros JM. Time to positivity of blood culture association with clinical presentation, prognosis and ESBL-production in Escherichia coli bacteremia. Eur J Clin Microbiol Infect Dis. 2012; 31:2191-5.

[5] McGowan KL, Foster JA, Coffin SE. Outpatient pediatric blood cultures: time to positivity. Pediatrics. 2000; 106:251-5.

[6] Brun-Buisson C, Doyon F, Carlet J, Dellamonica P, Gouin F, Lepoutre A, et al. Incidence, risk factors, and outcome of severe sepsis and septic shock in adults. A multicenter prospective study in intensive care units. French ICU Group for Severe Sepsis. JAMA. 1995; 274:968-74.

[7] Cornish N, Kirkley BA, Easley KA, Washington JA. Reassessment of the incubation time in a controlled clinical comparison of the BacT/Alert aerobic FAN bottle and standard anaerobic bottle used aerobically for the detection of bloodstream infections. Diagn Microbiol Infect Dis. 1998; 32:1-7.

[8] Doern GV, Brueggemann AB, Dunne WM, Jenkins SG, Halstead DC, McLaughlin JC. Four-day incubation period for blood culture bottles processed with the Difco ESP blood culture system. J Clin Microbiol. 1997; 35:1290-2.

[9] Han XY, Truant AL. The detection of positive blood cultures by the AccuMed ESP-384 system: the clinical significance of three-day testing. Diagn Microbiol Infect Dis. 1999; 33:1-6.

[10] Previsdomini M, Gini M, Cerutti B, Dolina M, Perren A. Predictors of positive blood cultures in critically ill patients: a retrospective evaluation. Croat Med J. 2012; 53:30-9.

[11] Reisner BS, Woods GL. Times to detection of bacteria and yeasts in BACTEC 9240 blood culture bottles. J Clin Microbiol. 1999; 37:2024-6.

[12] Towns ML, Jarvis WR, Hsueh PR. Guidelines on blood cultures. J Microbiol Immunol Infect. 2010; 43:347-9.

[13] Peleg AY, Seifert H, Paterson DL. Acinetobacter baumannii: emergence of a successful pathogen. Clin Microbiol Rev. 2008; 21:538-82.

[14] Hall KK, Lyman JA. Updated review of blood culture contamination. Clin Microbiol Rev. 2006; 19:788-802.

[15] Weinstein MP. Blood culture contamination: persisting problems and partial progress. J Clin Microbiol. 2003; 41:2275-8.

[16] Weinstein MP, Towns ML, Quartey SM, Mirrett S, Reimer LG, Parmigiani G, et al. The clinical significance of positive blood cultures in the 1990s: a prospective comprehensive evaluation of the microbiology, epidemiology, and outcome of bacteremia and fungemia in adults. Clin Infect Dis. 1997; 24:584-602.

[17] Weinstein MP, Doern GV. A critical apprasial of the role of the clinical microbiology laboratory in the diagnosis of bloodstream infections. J Clin Microbiol. 2011; 49:S26-9.

[18] Levy MM, Fink MP, Marshall JC, Abraham E, Angus D, Cook D, et al. 2001 SCCM/ESICM/ACCP/ATS/SIS international sepsis definitions conference. Crit Care Med. 2003; 31:1250-6.

[19] Barenfanger J, Drake C, Lawhorn J, Verhulst SJ. Comparison of chlorhexidine and tincture of iodine for skin antisepsis in preparation for blood sample collection. J Clin Microbiol. 2004; 42:2216-7.

[20] Calfee DP, Farr BM. Comparison of four antiseptic preparations for skin in the prevention of contamination of percutaneously drawn blood cultures: a randomized trial. J Clin Microbiol. 2002; 40:1660-5.

[21] Suwanpimolkul G, Pongkumpai M, Suankratay C. A randomized trial of $2 \%$ chlorhexidine tincture compared with $10 \%$ aqueous povidone-iodine for venipuncture site disinfection: Effects on blood culture contamination rates. J Infect. 2008; 56:354-9.

[22] Mimoz O, Karim A, Mercat A, Cosseron M, Falissard B, Parker F, et al. Chlorhexidine compared with povidone-iodine as skin preparation before blood culture. A randomized, controlled trial. Ann Intern Med. 1999; 131:834-7.

[23] Neuman MI, Harper MB. Time to positivity of blood cultures for children with Streptococcus pneumoniae bacteremia. Clin Infect Dis. 2001; 33:1324-8. 